\title{
Characterization of bortezomib-adapted I-45 mesothelioma cells
}

\author{
Lidong Zhang**1, James E Littlejohn'1, Yu Cui1,2, Xiaobo Cao', Chander Peddaboina' and W Roy Smythe*1
}

\begin{abstract}
Background: Bortezomib, a proteasome-specific inhibitor, has emerged as a promising cancer therapeutic agent. However, development of resistance to bortezomib may pose a challenge to effective anticancer therapy. Therefore, characterization of cellular mechanisms involved in bortezomib resistance and development of effective strategies to overcome this resistance represent important steps in the advancement of bortezomib-mediated cancer therapy.

Results: The present study reports the development of I-45-BTZ-R, a bortezomib-resistant cell line, from the bortezomib-sensitive mesothelioma cell line I-45. I-45-BTZ-R cells showed no cross-resistance to the chemotherapeutic drugs cisplatin, 5-fluorouracil, and doxorubicin. Moreover, the bortezomib-adapted I-45-BTZ-R cells had decreased growth kinemics and did not over express proteasome subunit $\beta 5$ (PSMB5) as compared to parental I-45 cells. I-45-BTZR cells and parental I-45 cells showed similar inhibition of proteasome activity, but I-45-BTZ-R cells exhibited much less accumulation of ubiquitinated proteins following exposure to $40 \mathrm{~nm}$ bortezomib. Further studies revealed that relatively low doses of bortezomib did not induce an unfolded protein response (UPR) in the bortezomib-adapted cells, while higher doses induced UPR with concomitant cell death, as evidenced by higher expression of the mitochondrial chaperone protein Bip and the endoplasmic reticulum (ER) stress-related pro-apoptotic protein CHOP. In addition, bortezomib exposure did not induce the accumulation of the pro-apoptotic proteins p53, Mcl-1S, and noxa in the bortezomib-adapted cells.
\end{abstract}

Conclusion: These results suggest that UPR evasion, together with reduced pro-apoptotic gene induction, accounts for bortezomib resistance in the bortezomib-adapted mesothelioma cell line I-45-BTZ-R.

\section{Background}

The $26 \mathrm{~S}$ proteasome is a multi-subunit enzymatic complex composed of a barrel-shaped $20 \mathrm{~S}$ core region with catalytic activity adjacent to a 195 regulatory complex [1]. Recent investigations have revealed that the ubiquitinproteasome pathway plays a key role in regulating the homeostasis of cellular proteins involved in cell cycle regulation, cell survival, and apoptosis. Therapeutic targeting of the proteasome pathway with the specific inhibitor bortezomib has been successful in selectively inducing apoptosis in mesothelioma and a variety of other human cancer cells, with tolerable toxicity to normal cells and tissues [2-4]. Importantly, bortezomib has received US FDA approval for the treatment of patients with multiple myeloma (MM) and mantle cell lymphoma [5].

* Correspondence: lidongz@bcm.tmc.edu, rsmythe@swmail.sw.org Section of Surgery Research, Department of Surgery, Texas A \& M University Health Science Center College of Medicine and Scott \& White Memorial Hospital, Temple, Texas, USA

Full list of author information is available at the end of the article
However, cancer cell resistance to bortezomib-mediated apoptosis may limit the successful application of bortezomib as a cancer therapeutic agent. Although bortezomib shows much stronger anti-tumor activity in MM than in solid tumors, approximately $50 \%$ of MMs do not respond to this medication [6,7]. Moreover, many patients with MM who initially responded to bortezomib ultimately relapse with bortezomib-refractory disease [8], suggesting that even in the cancer exhibiting the best treatment response, bortezomib resistance remains a significant obstacle to treatment efficacy.

Bortezomib (PS-341, or Velcade) is a dipeptidyl boronic acid that reversely inhibits $20 \mathrm{~S}$ proteasome activity. In $\mathrm{MM}$, the transcriptional regulatory protein nuclear factor $\kappa \mathrm{B}(\mathrm{NF} \kappa \mathrm{B})$ has been proposed as a major target of bortezomib $[4,9,10]$. Bortezomib blocks the degradation of ІкB, a cytoplasmic $\mathrm{NF} \kappa \mathrm{B}$ inhibitory protein, effectively reducing NFKB translocation from the cytoplasm to the nucleus and blocking its transcriptional regulatory activ- 
ity. Given the established roles of NFKB in angiogenesis, cell invasion, oncogenesis, proliferation, and inhibition of apoptosis, inhibition of this important transcription factor is widely regarded as an attractive strategy of cancer therapy and a primary mechanism of bortezomib antitumor activity in MM cells $[4,9,10]$. Moreover, as a proteasome inhibitor, bortezomib is able to overcome chemoresistance or induce chemosensitization by inhibiting the NFkB functions that are typically activated by conventional chemotherapeutic agents $[9,10]$. Beyond $\mathrm{NF} \kappa \mathrm{B}$ inhibition, bortezomib also induces the intracellular unfolded protein response (UPR) [11] and stabilizes the expression of the proapoptotic genes p53 [12], Bim [13], or noxa [14], indirectly contributing to bortezomib anti-tumor activity.

Although progress has made in defining bortezomib mechanisms of action, mechanisms of bortezomib resistance in cancer are not well understood. In one early study, heat shock protein 27 (HSP27) was shown to play an important role in bortezomib resistance [15]. Recently, evidence was reported supporting a relationship between proteasome subunit $\beta 5$ (PSMB5) expression and bortezomib resistance. Bortezomib is a reversible inhibitor that primarily targets PSMB5, which is responsible for the chymotrypsin activity of the $26 \mathrm{~S}$ proteasome. Several studies focused on acute myeloid leukemia, lymphoma, and MM have shown that a series of bortezomib-adapted cell lines developed from the above malignancies exhibit higher PSMB5 expression at the both RNA and protein levels than the respective parental bortezomib-sensitive cells [16-19]. Further investigation demonstrated that inhibition of PSMB5 expression partially restored bortezomib sensitivity in resistant cells [18].

In the present study, a novel bortezomib resistant cell line was developed from the mesothelioma cell line I-45. Our results suggest that UPR evasion together with reduced pro-apoptotic gene induction accounted for bortezomib resistance in this new bortezomib-adapted mesothelioma cell line.

\section{Results}

Development of the bortezomib-adapted mesothelioma cell line I-45-BTZ-R

To determine whether prolonged exposure of the mesothelioma cell line I- 45 to bortezomib would select for cells resistant to bortezomib treatment, I-45 cells were exposed to $12 \mathrm{nM}$ bortezomib (its $\mathrm{IC}_{50}$ concentration). The RPMI-1640 cell culture medium was changed every three days and fresh bortezomib was added at the same time. After one month of cell incubation at this concentration, the selection concentration was increased to 15 nM. Extracellular concentrations of bortezomib were increased in a step-wise fashion over a period of 6 months, culminating in a drug concentration of $40 \mathrm{nM}$.
Selection was halted at this dose, since $40 \mathrm{nM}$ is already higher than average plasma concentrations measured in patients following administration of therapeutically effective does of bortezomib. The Bortezomib-adapted mesothelioma cell line I-45-BTZ-R showed consistent resistance to bortezomib, as I-45-BTZ-R cells maintained the same degree of resistance after two months of culture in bortezomib-free medium. As is presented in Figure 1A, three days after treatment with different concentrations of bortezomib, I-45-BTZ-R cells showed much higher cell viability than parental I- 45 cells, with $\mathrm{IC}_{50}$ values of 185.5 $\pm 0.3 \mathrm{nM}$ and $12.1 \pm 0.4 \mathrm{nM}$, respectively. Cleavage of caspase-3 or PARP was used as an indicator of bortezomibinduced apoptosis. As seen in Figure 1B, one, two, or three days after cells of exposure to $40 \mathrm{nM}$ bortezomib, this agent induced caspase- 3 and PARP cleavage in parental I-45 cells, but not in I-45-BTZ-R cells. These data indicated that I-45-BTZ-R cells do not undergo apoptosis following bortezomib treatment. Cell cycle analysis of treatment-induced sub-G1 cell populations (Figure 1C) confirmed these results. As expected, bortezomib did not induce strong G2/M arrest in I-45-BTZ-R cells as compared with parental I- 45 cells (Figure 1C). 25 $\mathrm{nM}$ bortezomib treatment for 72 hours increased G2/M population to $70.9 \%$ from $4 \%$ of the basal level in parental I-45 cells. However, the same treatment even did not change G2/M phase population in I-45-BTZ-R cells, which remained $10.5 \%$ as compared to $10.1 \%$ of the basal level. With increase in treatment doses, bortezomib induced less G2/M arrest in I- 45 cells, which may indicate that more cells at G2/M phase went to apoptotic subG1 phase.

To determine cell growth kinetics, the same numbers of both cell types were maintained in culture for eight days. Interestingly, I-45-BTZ-R cells exhibited significantly reduced cell growth as compared to I-45 cells $(\mathrm{p}<0.05$ at day $2 ; \mathrm{p}<0.01$ at days 4,6 , and 8 ) (Figure 1D).

\section{Proteasome subunit PSMB5 protein expression and chymotrypsin-like activity of in I-45-BTZ-R and I-45 cells}

Several recent reports on acute myeloid leukemia, lymphoma, and MM described increased PSMB5 RNA and protein expression in bortezomib-adapted cells, and inhibition of PSMB5 expression partially restored bortezomib sensitivity in resistant cells, indicating that PSMB5 over expression is important for bortezomib resistance in cancer [16-19]. However, the present study did not detect a difference in PSMB5 protein expression between the two cell lines (Figure 2A). Moreover, expression of two other proteasome subunits, $\beta 1$ (PSMB1) and $\beta 2$ (PSMB2), which are responsible for caspase-like, or trypsin-like activity, respectively, was also not altered in the resistant cell line (Figure 2A). 

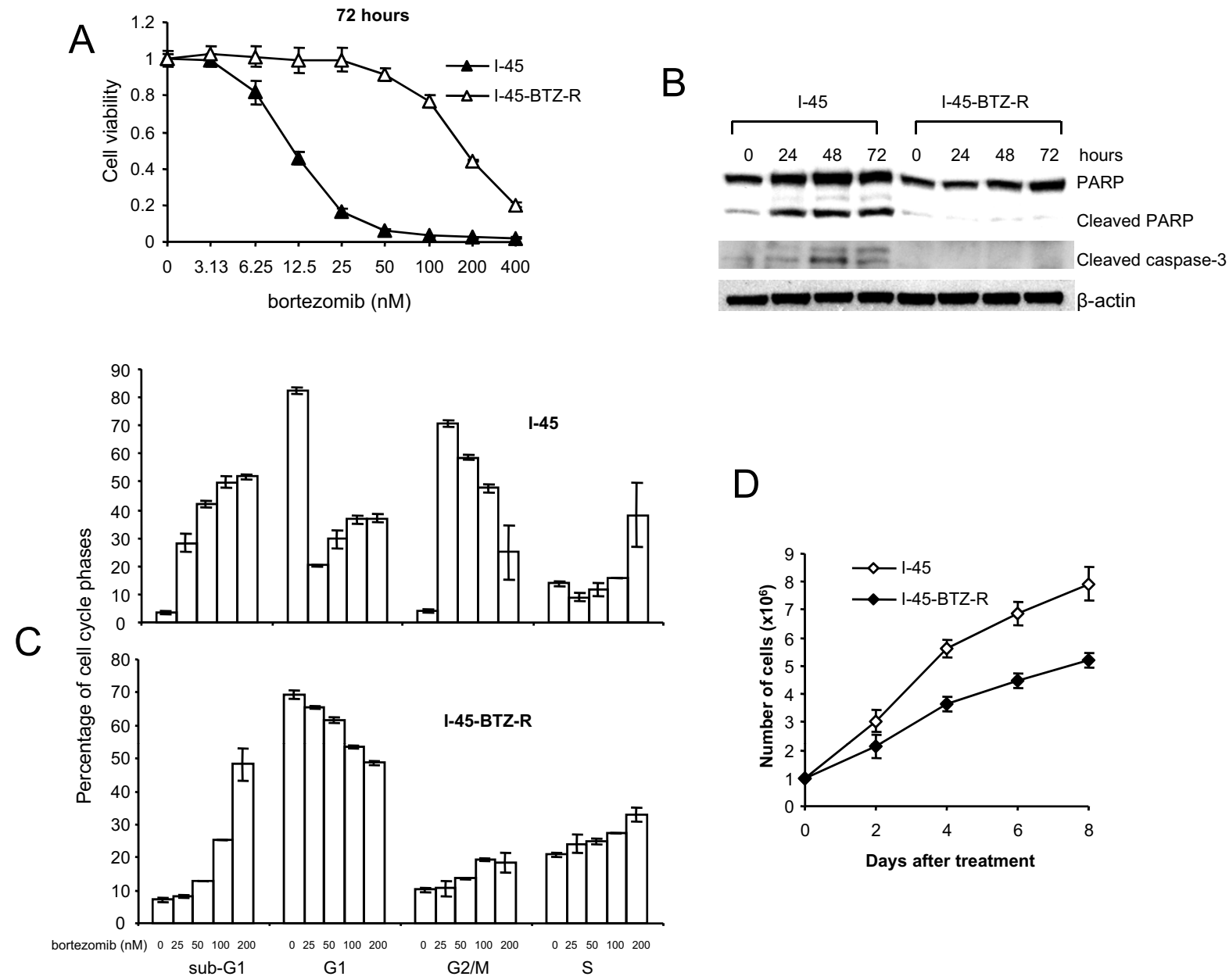

Figure 1 Characterization of the bortezomib-adapted cell line I-45-BTZ-R. A: I-45 and I-45-BTZ-R cells were treated with bortezomib (3.13 nM to $400 \mathrm{nM}$ ) for 72 hours. Cell viability was determined after treatment using the XTT assay. Control cells were treated with PBS and their viability was set as $100 \%$. Values are the mean \pm SD of triplicate assays from two experiments. B: $1-45$ and I-45-BTZ-R cells were treated with $40 \mathrm{nM}$ bortezomib for 24 , 48, or 72 hours. Caspase-3 and PARP activation (cleavage) were analyzed by Western blot analysis. C: I-45 and I-45-BTZ-R cells were treated with bortezomib ( $25 \mathrm{nM}$ to $200 \mathrm{nM}$ ) for 72 hours. Percentages of sub-G1 cells and cell cycle distribution were determined by flow cytometry analysis. Values are the mean \pm SD of two experiments. D: I-45 and I-45-BTZ-R cells were seeded into $20 \mathrm{~cm}$ cell culture dishes $\left(1 \times 10^{6}\right.$ cells per dish). At days $2,4,6$, or 8 , the cells were trypsinized and stained with trypan blue. Viable cells were counted under a microscope using a hemocytometer. Values are the mean \pm SD of three experiments.

Since bortezomib specifically inhibits proteasome chymotrypsin-like activity, we also evaluated proteasome chymotrypsin-like activity in parental and resistant cell lines using a substrate fluorescence assay. Although basal chymotrypsin-like activity was reduced approximately $30 \%$ in I-45-BTZ-R cells as compared to I- 45 cells, both cell lines maintained approximately 25 to $40 \%$ of their original proteasome activity following bortezomib treatment (Figure 2B). These data indicated that chymotrypsin-like activity can be inhibited by bortezomib not only in parental I-45 cells, but also in I-45-BTZ-R cells, and that the mechanisms underlying I-45-BTZ-R cell resistance to bortezomib may be independent of proteasome activity.
Characteristic features of proteasome inhibition include the accumulation of ubiquitinated proteins, which can induce ER stress and UPR, and stabilization of pro-apoptotic genes, leading to mitochondrial membrane potential changes and apoptosis [11]. To this end, we evaluated the expression of ubiquitinated proteins in both cell lines following bortezomib treatment. As is shown in Figure 2C, basal levels of ubiquitinated proteins were similar in both of the cell lines. However, the concentration of ubiquitinated proteins in I-45 cells increased sharply 24 hours after exposure to $40 \mathrm{nM}$ bortezomib, with further increases observed after 48 or 72 hours of drug exposure. Conversely, I-45-BTZ-R cells exhibited lower accumulation of ubiquitinated proteins after 24 


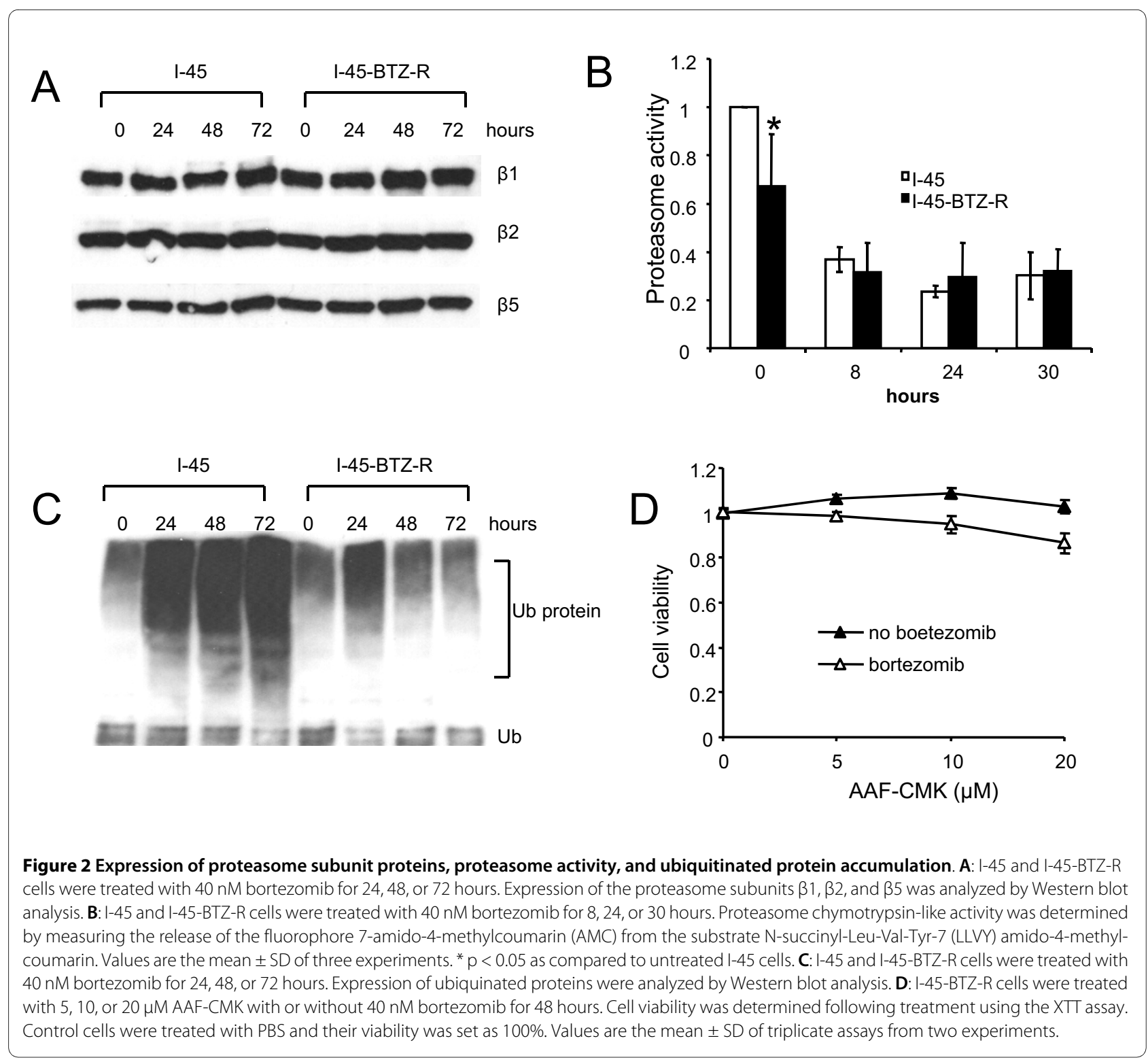

hours of bortezomib exposure, and the concentration of ubiquitinated proteins returned to the basal level following 48 or 72 hours.

Since I-45-BTZ-R cells survived yet maintained the same low proteasome activity and showed much less accumulation of ubiquitinated proteins as did I-45 cells in response to toxic doses of bortezomib, we hypothesized that alternate cellular protease pathways might have become activated, essentially compensating for reduced 26 proteasome activity in I-45-BTZ-R cells and reducing bortezomib toxicity. One candidate protease was tripeptidyl peptidase II (TPPII), which plays a critical role in cleaving proteasomal-produced peptides into tripeptides prior to further degradation by other peptidases. TPPII upregulation has been reported in some lymphoma cells resistant to the proteasome inhibitor tri-leucine-vinylsulphone (NLVS), and inhibition of TPPII function using the specific inhibitor AAF-CMK either directly induces apoptosis or indirectly induces NLVS-mediated cell death $[20,21]$. In the present study, TPPII expression was not evident in either I-45 or I-45-BTZ-R cells by Western blot analysis (data not shown). Moreover, treatment with AAF-CMK at 5,10 , or $20 \mu \mathrm{M}$ for 48 hours did not induce decreased I-45-BTZ-R cell viability (Figure 2D). Although combined treatment with AAF-CMK and bortezomib significantly decreased cell viability as compared with single agent treatment, cell viability remained very high $(>85 \%)$. These results suggest that TPPII did not compensate for reduced proteasome activity in I-45-BTZ-R cells. 


\section{Lack of cross-resistance to various anticancer drugs}

The cellular multi-drug resistance proteins MDR1 (p-glycoprotein) and MRP1 are critical transmembrane efflux pumps that transport various chemotherapeutic drugs out of cells. Both I-45 and I-45-BTZ-R cells showed no evidence of MDR1 or MRP1 by Western blot analysis (data not shown) and both cell lines demonstrated similar sensitivity to the chemotherapy drug cisplatin, which is commonly used to treat patients with mesothelioma (Figure 3). We also tested the responses of both cell lines to the chemotherapy drugs 5-fluorouracil and doxorubicin. As was observed with cisplatin, both cell lines showed similar sensitivity to treatment with 5-fluorouracil or doxorubicin (data not shown), indicating there the absence of cell line cross-resistance to common chemotherapy drugs.

\section{Bortezomib does not induce ER stress and UPR in I-45-BTZ- $R$ cells}

Recent studies demonstrated that bortezomib activates ER stress and induces UPR in cancer cells [11,22]. Since the mitochondrial chaperone protein Bip and the proapoptotic transcriptional factor $\mathrm{CHOP}$ are markers of $\mathrm{ER}$ stress, we evaluated Bip and CHOP protein expression in I-45 and I-45-BTZ-R cells by Western blot analysis. Results indicated that basal Bip expression was similar in both cell lines. However, treatment with $40 \mathrm{nM}$ bortezomib stimulated higher Bip and CHOP protein expression in I-45 cells but not in I-45-BTZ-R cells (Figure 4A). These results indicated that bortezomib treatment did not induce ER stress or UPR in I-45-BTZ-R cells. To

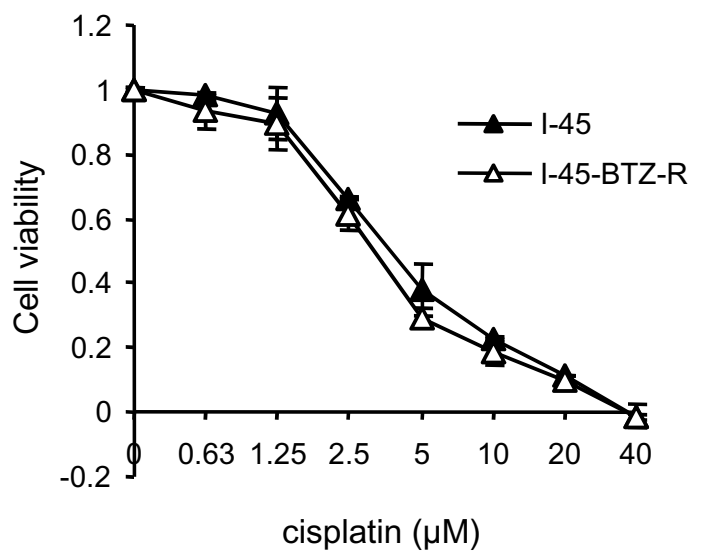

Figure 3 Sensitivity of I-45 and I-45-BTZ-R cells to cisplatin. I-45 and I-45-BTZ-R cells were treated with cisplatin $(0.63 \mu \mathrm{M}$ to $40 \mu \mathrm{M})$ for 72 hours. Cell viability was determined following treatment using the XTT assay. Control cells were treated with PBS and their viability was set as $100 \%$. Values are the mean \pm SD of triplicate assays from two experiments.

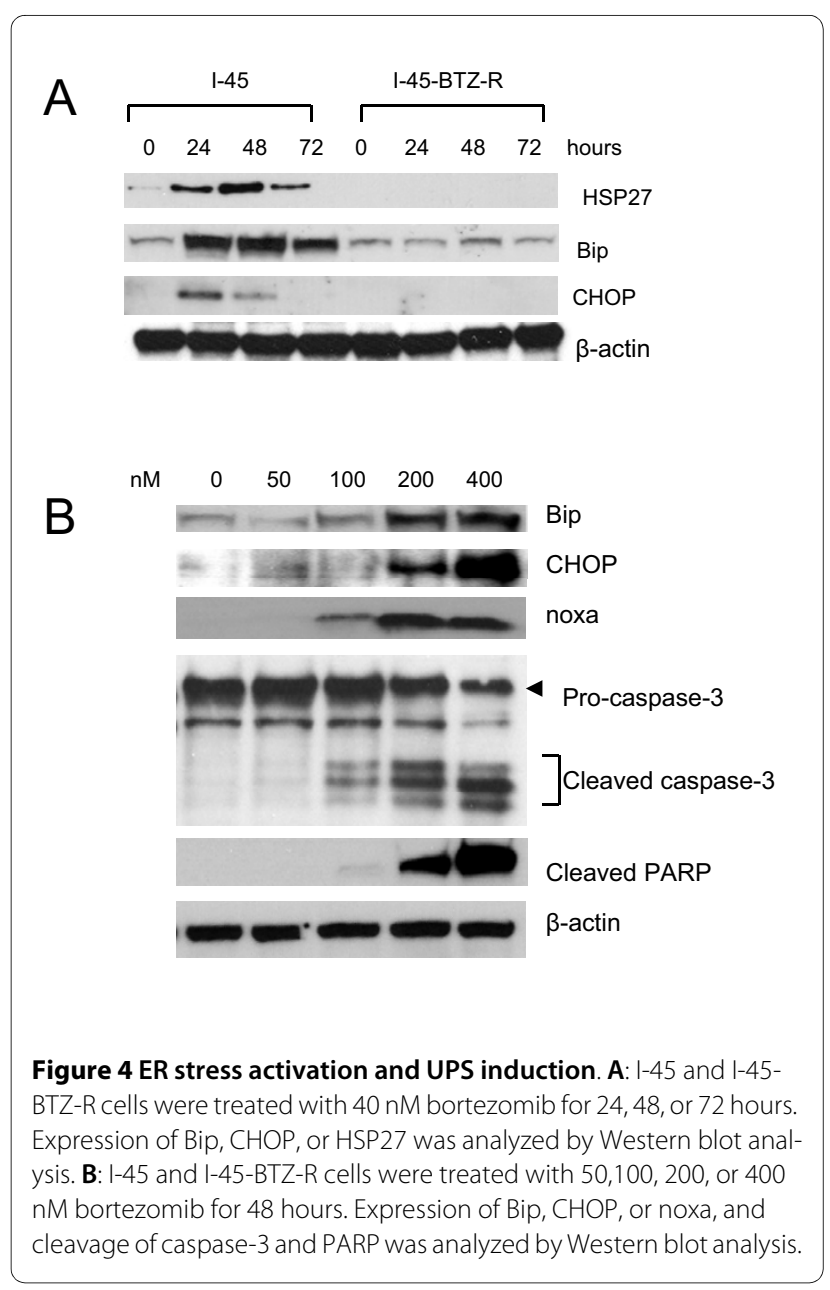

determine whether lethal concentrations of bortezomib could induce ER stress and UPR, I-45-BTZ-R cells were incubated with increasing concentrations of bortezomib $(40,100,200$, and $400 \mathrm{nM})$. As is shown in Figure 4B, bortezomib at $50 \mathrm{nM}$ did not induce expression of either Bip or CHOP. However, bortezomib at 100 to $400 \mathrm{nM}$ induced an increase in the expression of both Bip and $\mathrm{CHOP}$ in parallel with an increase in apoptosis, as evidenced by increased cleavage of capase- 3 and PARP.

Since CHOP is a pro-apoptotic transcriptional factor during ER stress and UPR, we tested whether siRNA knockdown of CHOP expression could protect I-45BTZ-R cells from bortezomib-induced cell death. Transfection with $100 \mathrm{nM}$ of CHOP siRNAs for 48 hours followed by treatment with $200 \mathrm{nM}$ bortezomib for an additional 72 hours effectively reduced bortezomibmediated CHOP protein expression (Figure 5A). Knockdown of CHOP gene expression also protected I-45-BTZ$\mathrm{R}$ cells from bortezomib-mediated apoptosis, as evidenced by a significant increase in cell viability from 53.9 $\pm 5.4 \%$ to $72.3 \pm 2.6 \%$ in cells treated with in control siRNA versus $\mathrm{CHOP}$-specific siRNA, respectively (Figure 


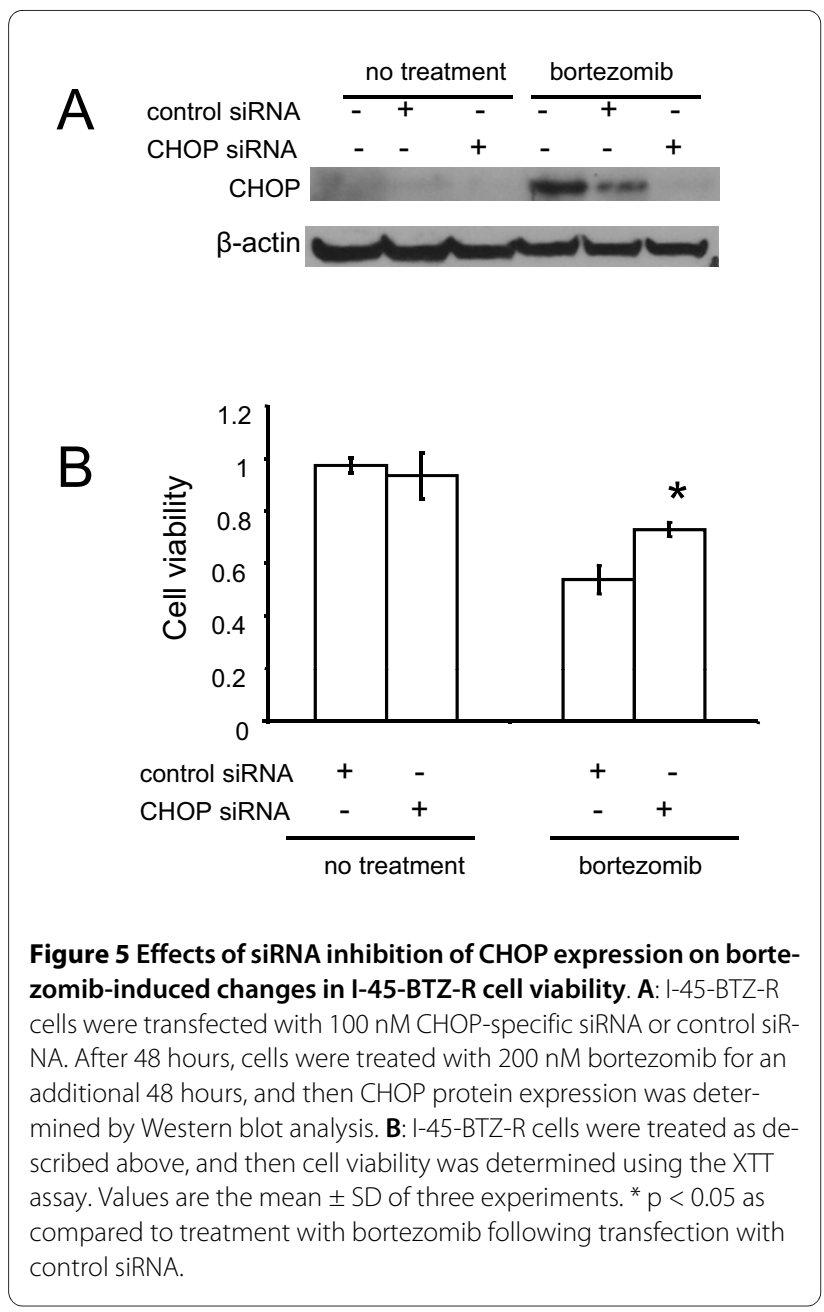

5B). In addition, our data also demonstrated that siRNAmediated knockdown of CHOP gene expression also partially protected parental I-45 cells from bortezomibinduced cell death (data not shown).

\section{Bortezomib induces noxa protein expression in I-45 cells but not in I-45-BTZ-R cells}

To better understand the molecular mechanisms underlying bortezomib resistance in I-45-BTZ-R cells, we examined $\mathrm{BCl}-2$ family member protein expression by Western blot analysis. Results indicated that several Bcl-2 family proteins showed altered protein expression following bortezomib treatment (Figure 6). First, $\mathrm{Bcl}-\mathrm{x}_{\mathrm{L}}$ protein expression increased slightly from 24 to 72 hours after treatment with $40 \mathrm{nM}$ bortezomib in both of the cell lines, which may not account for bortezomib resistance in I-45-BTZ-R cells. Second, bortezomib treatment increased expression of the anti-apoptotic protein Mcl$1 \mathrm{~L}$ in I- 45 cells approximately $100 \%$ at 24,48 , or 72 hours, while expression of the pro-apoptotic protein Mcl-1S was increased approximately $150 \%$ at 24 hours, but then decreased at 48 and 72 hours, Pro-apoptotic Mcl-1S is

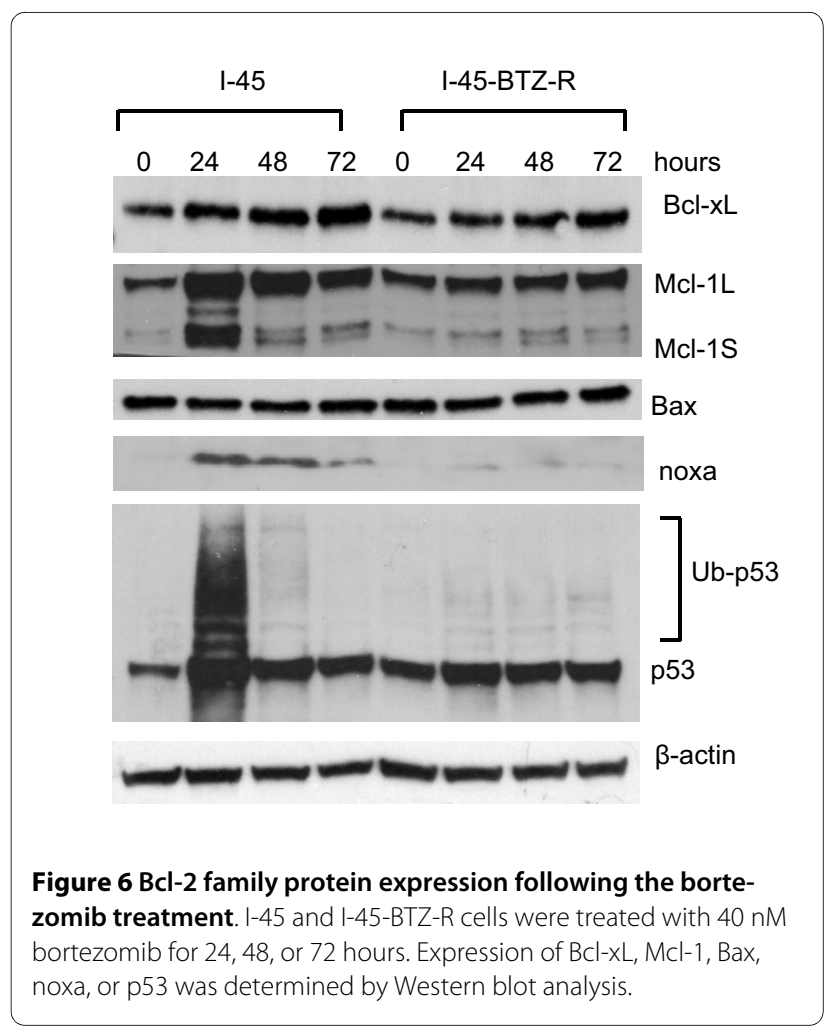

reported to be a $\mathrm{BH} 3$ domain-only protein capable of dimerizing with Mcl-1L, thus reducing mcl-1L anti-apoptotic function [23]. Different results were observed in in I-45-BTZ-R cells, where bortezomib treatment slightly increased Mcl-1L expression over time, but no change in Mcl-1S expression was detected.

Strong expression of the noxa protein was also detected in I-45 cells after treatment with bortezomib, while noxa expression was barely detectable in I-45-BTZ-R cells subjected to the same treatment (Figure 6). Impressively, bortezomib at 100 to $400 \mathrm{nM}$ also induced strong noxa expression in I-45-BTZ-R cells (Figure 4B), suggesting that noxa expression was correlated with bortezomibinduced cell death.

In addition, $40 \mathrm{nM}$ bortezomib treatment induced strong p53 protein accumulation in parental I- 45 cells, but only week $\mathrm{p} 53$ protein accumulation was observed in I-45-BTZ-R cells following bortezomib exposure (Figure 6).

\section{Discussion}

In the present study, we developed a bortezomib-resistant cell line (I-45-BTZ-R) from a bortezomib-sensitive mesothelioma cell line (I-45). I-45-BTZ-R cells showed no cross-resistance with the common chemotherapeutic drugs cisplatin, 5-fluorouracil, and doxorubicin. Moreover, we observed that the bortezomib-adapted I-45BTZ-R cells exhibited decreased growth kinemics as 
compared to the parental I- 45 cells. We also found that I45-BTZ-R cells did not over express the proteasome subunit PSMB5 as compared with the parental I-45 cells. In addition, $40 \mathrm{nM}$ bortezomib induced similar inhibition of proteasome activity in the bortezomib-adapted cells and the parental I- 45 cells, but significantly reduced accumulation of ubiquitinated protein accumulation. Further studies revealed that relatively low doses of bortezomib did not induce UPR in the bortezomib-adapted cells, while higher doses induced UPR with concomitant cell death, as evidenced by higher protein expression of the mitochondrial chaperone Bip and the ER stress-related pro-apoptotic gene CHOP. Bortezomib treatment of I-45BTZ-R cells also failed to induce the accumulation of the pro-apoptotic genes p53, Mcl-1S, and noxa. These results suggest that evading UPR together with reduced induction of pro-apoptotic gene expression accounts for bortezomib resistance in these bortezomib-adapted mesothelioma cells.

Bortezomib is a reversible proteasome inhibitor that primarily targets the PSMB5 subunit, which is responsible for $26 \mathrm{~S}$ proteasome chymotrypsin activity. Several research groups have recently developed bortezomibresistant cells representing different type of cancer, including acute myeloid leukemia, lymphoma, and MM [16-19]. Most of the reported bortezomib-adapted cells have shown higher PSMB5 RNA and protein expression as compared to the respective parental bortezomib-sensitive cells. A missense point mutation has been reported in a highly conserved PSMB5 bortezomib-binding pocket and that siRNA-mediated reduction of PSMB5 expression restored bortezomib sensitivity in the bortezomibresistant cell line [18]. In line with higher PSMB5 expression, most reported bortezomib-adapted cells showed increased proteasome activity as compared to their respective parental cell lines [16-19]. This increased activity has been used as a basis for explaining increased cell survival following a lethal challenge with proteasome inhibition. In the present study, over expression of proteasome subunit PSMB5 was not observed. We also did not find any mutations by DNA sequencing in the coding region of PSMB5 in both of the cell lines (data not shown). Accordingly, the bortezomib-adapted cells and I45 cells showed the same degree of bortezomib-induced proteasome inhibition. I-45-BTZ-R cells showed much less accumulation of ubiquitinated proteins following bortezomib treatment. There are two possible explanations for this observation. First, an alternate protease pathway may compensate for reduced proteasome function. For example, in lymphoma, continuous inhibition of proteasome activity selected for proteasome inhibitorresistant cells with lower proteasome activity, but higher expression of TPPII, which effectively replaced certain proteasome functions [20,21]. However, TPPII was not upregulated in I-45-BTZ-R cells (data not shown) and inhibition of TPPII activity using the specific inhibitor AAF-cmk had very little effect on the sensitivity of I-45BTZ-R cells to bortezomib (Figure 2D). However, we could not exclude the possibility that the activity of other, as-yet unidentified proteases compensated for reduced proteasome function in I-45-BTZ-R cells. Second, the slower growth of I-45-BTZ-R cells may have induced a general decrease in protein synthesis, resulting in a reduction in the number of ubiquitinated proteins. This possibility may also partially explain our observation that relatively low concentrations of bortezomib did not induce ER stress and UPR in I-45-BTZ-R cells.

The ER is a eukaryotic organelle critical to the production and modification of one third of all cellular proteins. In the ER lumen, excessive accumulation of misfolded or oxidized proteins induced by ER stress leads to induction of the UPR, a protective mechanism that initially restores the luminal folding capacity of the ER, but will ultimately trigger cell death if the protective mechanism is overwhelmed. During ER stress, increased concentrations of protein chaperones, particularly Bip, can limit protein aggregation inside the ER and inhibit general protein synthesis in order to reduce the ER-Golgi network workload and the cellular damage induced by ER stress [22,24,25]. CHOP, another protein marker of ER stress, functions to mediate the execution of programmed cell death $[22,24,25]$. It has been reported that bortezomib induces apoptosis in MM [11] and head and neck squamous carcinoma cells [26] by activating ER stress concurrent with upregulation of Bip and CHOP. In the present study, we observed upregulation of Bip and CHOP in the bortezomib-sensitive cell line I- 45 following bortezomib treatment, indicating bortezomib was able to induce ER stress in this mesothelioma cell line. However, low doses of bortezomib did not induce ER stress and apoptosis in the bortezomib-resistant cell line I-45-BTZ-R. Since proteasome inhibition resulting in the accumulation of ubiquitinated proteins is thought to induce ER stress and the UPR, reduced accumulation of ubiquitinated proteins in I-45-BTZ-R cells may have prevented ER stress induction and UPR-mediated cell death. Reduced accumulation of ubiquitinated proteins may have also decreased the stabilization of the pro-apoptotic proteins p53, Mcl-1S, and noxa in I-45-BTZ-R cells, thus further limiting bortezomib-induced apoptosis.

In agreement with the results of the present study, most other reported bortezomib-adapted cells did not exhibit much cross-resistance to most chemotherapeutic drugs $[16,18]$. These observations indicate that cancer cell resistance to bortezomib treatment can be overcome by most other therapies. Therefore, even though combination 
therapy using bortezomib together with chemotherapeutics does not show synergistic efficacy, such therapeutic approaches may still benefit patients through a reduction in the development of bortezomib resistance. Although expression of the multi-drug resistance proteins MDR1 and MRP1 was not detected in either I-45 or I-45-BTZ-R cell lines, we demonstrated that bortezomib can enter I45-BTZ-R cells and access to the proteasome complex through direct assessment of proteasome activity. I-45BTZ-R cells, which exhibit an approximately $30 \%$ decrease in basal proteasome activity as compared to I-45 cells, showed the same degree of proteasome inhibition following bortezomib treatment at doses that readily killed I-45-cells but spared I-45-BTZ-R cells.

\section{Conclusions}

The present study provides evidence supporting a new mechanism of bortezomib resistance that is independent of proteasome subunit PSMB5 expression and proteasome activity. In I-45-BTZ-R cells, reduced accumulation of ubiquitinated proteins prevents bortezomib induction of ER stress and the UPR and reduces the stabilization of the pro-apoptotic proteins $\mathrm{p} 53, \mathrm{Mcl}-1 \mathrm{~S}$, and noxa. These changes may account for bortezomib resistance in this cell line.

\section{Methods}

\section{Cell Culture and Reagents}

The human sarcomatoid type mesothelioma cell line I-45 expressing wild type p53 was kindly provided by Dr. J. Testa (Fox Chase Institute, Philadelphia, PA). Cells were grown in RPMI 1640 medium supplemented with $10 \%$ fetal bovine serum, glutamine, and antibiotics. Cells were cultured at $37^{\circ} \mathrm{C}$ in a humidified incubator containing a $5 \% \mathrm{CO}_{2}$ atmosphere. Bortezomib was kindly provided by Millennium: The Takeda Oncology Company (Cambridge, MA) and was dissolved in phosphate buffered saline (PBS) to make a stock concentration of $100 \mu \mathrm{M}$.

\section{Cell Viability Assay}

Cells were seeded at a density of 5000 cells/well in 96-well plates one day before exposure to various treatments. Following treatment, cell viability was determined using an XTT cell viability assay (Cell Proliferation Kit II, Roche Molecular Biochemicals, Indianapolis, IN) according to the manufacturer's protocol and as previously described [27].

\section{Cell Growth Assay}

Cells were seeded in $20 \mathrm{~cm}$ cell culture dishes at a density of $1 \times 10^{6}$ cells per dish. Cells were trypsinized at days 2 , 4,6 , or 8 and stained with trypan blue. Viable cells were counted under a microscope using a hemocytometer.

\section{Western Blot Analysis}

Western blot analysis was performed as described previously [27]. Rabbit polyclonal antibodies against PARP, caspase-3, Bcl- $\mathrm{L}_{\mathrm{L}}$, Bax, Bip, HSP27, or CHOP were purchased from Cell Signaling (Beverly, MA). Rabbit polyclonal anti-human p53 and Mcl-1 antibodies were provided by Santa Cruz Biotechnology (Santa Cruz, CA). A mouse monoclonal anti-human noxa antibody was purchased from Calbiochem (San Diego, CA). Mouse monoclonal antibodies against human $20 \mathrm{~S}$ proteasome subunits $\beta 1, \beta 2, \beta 5$ and anti-ubiquitin (FK2H) antibodies were obtained from Biomol (Plymouth Meeting, PA).

\section{Small Interfering RNA Transfection}

CHOP expression was silenced using a pool of four small interfering RNAs (siRNAs) directed against CHOP mRNA (Dharmacon, Lafayette, CO). Cells were transfected with $100 \mathrm{nM}$ of CHOP-specific siRNA or siCONTROL Non-Targeting Pool siRNAs (Dharmacon) using the transfection reagent Dharma FECT 1 (Dharmacon) according to the manufacturer's protocol. Cells were cultured for 48 hours, and then treated simultaneously with bortezomib for an additional 72 hours.

\section{Flow Cytometry}

Cells were trypsinized, washed once with cold PBS, and then fixed with $70 \%$ ethanol overnight at $4^{\circ} \mathrm{C}$. Fixed cells were suspended in PBS containing $25 \mu \mathrm{g} / \mathrm{mL}$ propidium iodide (Roche Diagnostics, Indianapolis, IN) and $10 \mu \mathrm{g} /$ $\mathrm{mL}$ RNase A (Sigma-Aldrich, St. Louis, MO) at $3^{\circ} \mathrm{C}$ for 30 minutes. Flow cytometry analysis for cell cycle distribution and determination of the sub-G1 apoptotic cell population was performed as previously described [27].

\section{Proteasome chymotrypsin-like activity assay}

Cells treated with bortezomib and untreated control cells were lysed in $20 \mathrm{mM}$ Tris- $\mathrm{HCl}$ buffer (pH7.6) by repeated freezing in liquid nitrogen and thawing in a $37^{\circ} \mathrm{C}$ water bath. Cell lysate chymotrypsin-like activity was determined by measuring the release of the fluorophore 7amido-4-methylcoumarin (AMC) from $10 \mu \mathrm{M}$ of the substrate N-succinyl-Leu-Val-Tyr-7 (LLVY) amido-4-methylcoumarin (Sigma-Aldrich). Fluorescence was measured on a Flexstation microplate fluorometer (Molecular Devices, Sunnyvale, CA, USA) at excitation/emission wavelengths of $380 / 440 \mathrm{~nm}$.

\section{Statistical Analysis}

Differences among treatment groups were assessed by analysis of variance using PRISM 4 software. $P$ values of $=$ 0.05 were regarded as significant. 


\section{List of Abbreviations}

UPR: unfolded protein response; ER: endoplasmic reticulum; NFкB: Nuclear factor кB; MM: multiple myeloma; PSMB5: proteasome subunit $\beta 5$; PSMB1: proteasome subunit $\beta 1$; PSMB2: proteasome subunit $\beta 2$; HSP27: heat shock protein 27; PBS: phosphate buffered saline; TPPII: tripeptidyl peptidase; NLVS: tri-leucine-vinyl-sulphone.

\section{Competing interests}

The authors declare that they have no competing interests.

\section{Authors' contributions}

LZ and WRS conceived the study, coordinated its design and execution, and drafted the manuscript. LZ, CP and YC performed the cell culture, cell viability assays, immunoblots and siRNA assays. XC and JEL were involved in the overall design of the study and helped draft the manuscript. All authors read and approved the final manuscript.

\section{Acknowledgements}

We would like to thank Glen Cryer in Scientific Publications for editorial review.

\section{Author Details}

'Section of Surgery Research, Department of Surgery, Texas A \& M University Health Science Center College of Medicine and Scott \& White Memorial Hospital, Temple, Texas, USA and 2Department of Oncology, Tianjin Union Medicine Center, Tianjin, China

Received: 19 November 2009 Accepted: 18 May 2010 Published: 18 May 2010

\section{References}

1. Adams J: The proteasome: a suitable antineoplastic target. Nat Rev Cancer 2004, 4(5):349-360

2. Adams J: The development of proteasome inhibitors as anticancer drugs. Cancer Cell 2004, 5(5):417-421.

3. Sanchez-Serrano I: Success in translational research: lessons from the development of bortezomib. Nat Rev Drug Discov 2006, 5(2):107-114.

4. Sartore-Bianchi A, Gasparri F, Galvani A, Nici L, Darnowski JW, Barbone D, Fennell DA, Gaudino G, Porta C, Mutti L: Bortezomib inhibits nuclear factor-kappaB dependent survival and has potent in vivo activity in mesothelioma. Clin Cancer Res 2007, 13(19):5942-5951.

5. Orlowski RZ, Kuhn DJ: Proteasome inhibitors in cancer therapy: lessons from the first decade. Clin Cancer Res 2008, 14(6):1649-1657.

6. Richardson PG, Barlogie B, Berenson J, Singhal S, Jagannath S, Irwin D, Rajkumar SV, Srkalovic G, Alsina M, Alexanian R, Siegel D, Orlowski RZ, Kuter D, Limentani SA, Lee S, Hideshima T, Esseltine DL, Kauffman M, Adams J, Schenkein DP, Anderson KC: A phase 2 study of bortezomib in relapsed, refractory myeloma. N Engl J Med 2003, 348(26):2609-2617.

7. Richardson PG, Sonneveld P, Schuster M, Irwin D, Stadtmauer E, Facon T, Harousseau JL, Ben-Yehuda D, Lonial S, Goldschmidt H, Reece D, Miguel JS, Blade J, Boccadoro M, Cavenagh J, Alsina M, Rajkumar SV, Lacy M, Jakubowiak A, Dalton W, Boral A, Esseltine DL, Schenkein D, Anderson KC: Extended follow-up of a phase 3 trial in relapsed multiple myeloma: final time-to-event results of the APEX trial. Blood 2007, 110(10):3557-3560.

8. Conner TM, Doan QD, Walters IB, LeBlanc AL, Beveridge RA: An observational, retrospective analysis of retreatment with bortezomib for multiple myeloma. Clin Lymphoma Myeloma 2008, 8(3):140-145.

9. Hideshima T, Richardson P, Chauhan D, Palombella VJ, Elliott PJ, Adams J, Anderson KC: The proteasome inhibitor PS-341 inhibits growth, induces apoptosis, and overcomes drug resistance in human multiple myeloma cells. Cancer Res 2001, 61(7):3071-3076.

10. Hideshima T, Chauhan D, Richardson P, Mitsiades C, Mitsiades N, Hayash T, Munshi N, Dang L, Castro A, Palombella V, Adams J, Anderson KC: NFkappa B as a therapeutic target in multiple myeloma. J Biol Chem 2002, 277(19):16639-16647.

11. Obeng EA, Carlson LM, Gutman DM, Harrington WJ Jr, Lee KP, Boise LH: Proteasome inhibitors induce a terminal unfolded protein response in multiple myeloma cells. Blood 2006, 107(12):4907-4916.
12. Williams SA, McConkey DJ: The proteasome inhibitor bortezomib stabilizes a novel active form of p53 in human LNCaP-Pro5 prostate cancer cells. Cancer Res 2003, 63(21):7338-7344.

13. Li C, Li R, Grandis JR, Johnson DE: Bortezomib induces apoptosis via Bim and Bik up-regulation and synergizes with cisplatin in the killing of head and neck squamous cell carcinoma cells. Mol Cancer Ther 2008, 7(6):1647-1655.

14. Qin JZ, Ziffra J, Stennett L, Bodner B, Bonish BK, Chaturvedi V, Bennett F, Pollock PM, Trent JM, Hendrix MJ, Rizzo P, Miele L, Nickoloff BJ: Proteasome inhibitors trigger NOXA-mediated apoptosis in melanoma and myeloma cells. Cancer Res 2005, 65(14):6282-6293.

15. Chauhan D, Li G, Shringarpure R, Podar K, Ohtake Y, Hideshima T, Anderson KC: Blockade of Hsp27 overcomes Bortezomib/proteasome inhibitor PS-341 resistance in lymphoma cells. Cancer Res 2003, 63(19):6174-6177.

16. Fuchs D, Berges C, Opelz G, Daniel V, Naujokat C: Increased expression and altered subunit composition of proteasomes induced by continuous proteasome inhibition establish apoptosis resistance and hyperproliferation of Burkitt lymphoma cells. J Cell Biochem 2008, 103(1):270-283.

17. Lu S, Chen Z, Yang J, Chen L, Gong S, Zhou H, Guo L, Wang J: Overexpression of the PSMB5 gene contributes to bortezomib resistance in T-lymphoblastic lymphoma/leukemia cells derived from Jurkat line. Exp Hemato/ 2008, 36(10):1278-1284

18. Oerlemans R, Franke NE, Assaraf YG, Cloos J, van ZI, Berkers CR, Scheffer GL, Debipersad K, Vojtekova K, Lemos C, Heijden JW van der, Ylstra B, Peters GJ, Kaspers GL, Dijkmans BA, Scheper RJ, Jansen G: Molecular basis of bortezomib resistance: proteasome subunit beta5 (PSMB5) gene mutation and overexpression of PSMB5 protein. Blood 2008, 112(6):2489-2499.

19. Ruckrich T, Kraus M, Gogel J, Beck A, Ovaa H, Verdoes M, Overkleeft HS, Kalbacher H, Driessen C: Characterization of the ubiquitin-proteasome system in bortezomib-adapted cells. Leukemia 2009, 23(6):1098-1105.

20. Gavioli R, Frisan T, Vertuani S, Bornkamm GW, Masucci MG: c-myc overexpression activates alternative pathways for intracellular proteolysis in lymphoma cells. Nat Cell Biol 2001, 3(3):283-288.

21. Glas R, Bogyo M, McMaster JS, Gaczynska M, Ploegh HL: A proteolytic system that compensates for loss of proteasome function. Nature 1998, 392(6676):618-622.

22. Fribley $A$, Wang $C Y$ : Proteasome inhibitor induces apoptosis through induction of endoplasmic reticulum stress. Cancer Biol Ther 2006, 5(7):745-748.

23. Bae J, Leo CP, Hsu SY, Hsueh AJ: MCL-1S, a splicing variant of the antiapoptotic $B C L-2$ family member $M C L-1$, encodes a proapoptotic protein possessing only the $\mathrm{BH} 3$ domain. J Biol Chem 2000, 275(33):25255-25261

24. Boyce M, Yuan J: Cellular response to endoplasmic reticulum stress: a matter of life or death. Cell Death Differ 2006, 13(3):363-373.

25. McConkey DJ, Zhu K: Mechanisms of proteasome inhibitor action and resistance in cancer. Drug Resist Updat 2008, 11(4-5):164-179.

26. Fribley A, Zeng $Q$, Wang CY: Proteasome inhibitor PS-341 induces apoptosis through induction of endoplasmic reticulum stress-reactive oxygen species in head and neck squamous cell carcinoma cells. $\mathrm{Mol}$ Cell Biol 2004, 24(22):9695-9704.

27. Zhang L, Gu J, Lin T, Huang X, Roth JA, Fang B: Mechanisms involved in development of resistance to adenovirus-mediated proapoptotic gene therapy in DLD1 human colon cancer cell line. Gene Ther 2002, 9(18):1262-1270.

doi: $10.1186 / 1476-4598-9-110$

Cite this article as: Zhang et al., Characterization of bortezomib-adapted I45 mesothelioma cells Molecular Cancer 2010, 9:110 